\title{
Chinese Formula Ganji Fang Inhibits Tumor Growth Through Regulating Cell Mitosis by Reducing ARPP- 19 Expression in Hepatocellular Carcinoma
}

\section{Chunlei Zhang}

Shanghai University of Traditional Chinese Medicine

\section{Kaiyue Tang}

Shanghai University of Traditonal Chinese Medicine

\section{Lili Yang}

Shanghai University of Traditional Chinese Medicine

Yang Liu

Shanghai University of Traditional Chinese Medicine

Yifan Zhang

Shanghai University of Traditional Chinese Medicine

Jinzu Yang

Shanghai University of Traditional Chinese Medicine

\section{Peiyong Zheng}

Shanghai University of Traditional Chinese Medicine

Haiyan Song ( $\sim$ songhy@126.com )

Shanghai University of Traditional Chinese Medicine https://orcid.org/0000-0003-2155-8110

\section{Research}

Keywords: Hepatocellular carcinoma, Ganji Fang, ARPP-19, Mitosis

Posted Date: June 10th, 2020

DOI: https://doi.org/10.21203/rs.3.rs-34059/v1

License: (1) (1) This work is licensed under a Creative Commons Attribution 4.0 International License. Read Full License 


\section{Abstract}

Background: Hepatocellular carcinoma (HCC) is one of the most common malignancies with high rate of mortality worldwide. Rare effective treatment is available for HCC patients especially at advanced stage. Ganji Fang (GJF), one formula of traditional Chinese medicine (TCM), has shown therapeutic effect on HCC in clinical application. This study aims at evaluating the anti-HCC effect and safety of GJF treatment, as well as exploring the potential underlying molecular mechanism by using HCC mouse model.

Methods: HepG2 cells were subcutaneously injected into the upper flank of male BALB/c nude mice to establish the HCC Xenograft model. Mice were randomly assigned to three groups, treated respectively with high (GJF-H group) or low dose (GJF-L group) of GJF or vehicle (Control group) via gavage. The tumor volume was detected dynamically. Four weeks later, the weight and proliferation activity of tumors were measured. Western blot and immunohistochemistry were used to detect the expression of cAMPregulated phosphoprotein 19 (ARPP-19) and the regulated molecules. H\&E staining of tissue section of liver and kidney was used to assess the toxicity of the formula. Foe investigation of survival time, SMMC 7721 cells were injected subcutaneously to the BALB/c-nude mice. The tumor-bearing mice were allocated into two groups (Control and GJF-H) and treated as above description. The death number of mice was recorded and the survival time was analyzed.

Results: Compared with control, the body weight and activity of GJF-treated mice were improved obviously. No toxic change was observed in tissues of liver and kidney. The tumor volume and weight was significantly down-regulated by GJF dose-dependently. The mice with GJF treatment showed the tendency of prolonging survival time. The proliferation index and PCNA expression in the tumor of GJF group were lower than in control. Furthermore, GJF down-regulated levels of ARPP-19 and phospho-(Ser) CDKs substrates, up-regulated level of inactivated cyclin division cycle 2 (Cdc2).

Conclusion: GJF can effectively inhibit tumor growth of $\mathrm{HCC}$ and improve the general condition of the nude mouse xenograft model. The mechanism of inhibiting tumor growth is partially related to downregulating ARPP-19 to inhibit mitosis and cell proliferation of HCC.

\section{Background}

Primary liver cancer is one of the most common malignancies worldwide. Hepatocellular carcinoma (HCC) accounts for about $80 \%-90 \%$ of primary liver cancers and is the second leading cause of cancerrelated deaths due to diagnostic delay and rapid progression[1-4]. Due to the high metastasis and relapse rate of $\mathrm{HCC}$, only $10 \%$ patients exist during the first five years following diagnosis[5]. Curative treatment for HCC, for instance, surgical resection and orthotopic liver transplantation, is only applicable to patients with small tumors [6, 7]. Systemic chemotherapy is one of the important methods. The disease control rate (DCR) of multikinase inhibitor sorafenib treatment was proved to be $19 \%$, and the drug only provides less than 3-month benefit of overall survival for patients with advanced HCC [8]. The effective percentage 
of chemotherapy with Adriamycin, 5-fluorouracil, interferon a and platinumbased combinations was confirmed to be approximately $20 \%$. The survival benefit of chemotherapy was not proved [9]. Additionally, targeted drug and systemic chemotherapy often have some side-effects, affecting the life quality of patients $[10,11]$.

Traditional Chinese medicine (TCM) has been used to treat malignant tumor for hundreds of years. Recent studies have indicated that TCM can induce cell-cycle arrest and attenuate the tumor-associated macrophage-stimulated proliferation to inhibit tumor proliferation [12-14]. In addition, more and more researches confirmed the synergism and detoxification of combination of TCM with chemo- or radiotherapy[15]. Yu et al. approved that bu-zhong-yi-qi-tang in combination with cisplatin can effectively reverse cisplatin resistance via inducing apoptosis and autophagy in lung cancer cells[16]. Xiao-chai-hutang could inhibit cell proliferation and induce apoptosis of HCC cells by regulating the expression of Bax, Bcl-2, CDK4 and cyclin-D1[17]. Studies have shown that Songyou Yin (SYY) combined with oxaliplatin can inhibit epithelial-mesenchymal transition (EMT) so that decrease the metastatic potential of the residual HCC after oxaliplatin treatment [18]. Further study showed SYY could regulate EMT through down-regulation of TGF- $\beta 1$ expression and inhibition of the SMAD2/3 signaling pathway[19]. The compound PHY906 is the extract of the classical TCM formula Huang-qin-tang (HQT). It composed of Scutellaria baicalensis Georgi, Paeonia lactiflora Pall, Glycyrrhiza uralensis Fisch and Ziziphus jujuba Mill [15]. PHY906 combined with some anticancer drugs have synergistic effects in cancer therapy. The toxicity and side effect of chemotherapy could be significantly alleviated by PHY906 treatment[20]. It can be used for the adjuvant of antineoplastics (Sorafenib, CTP-11, 5-FU, leucovorin (LV) and capecitabine) in the treatment of advanced colorectal, pancreatic, and HCC[21, 22].

Ganji Fang (GJF) is an empirical formula used for tumor treatment in Longhua hospital for decades. Clinical investigations have shown that GJF could relieve tumor growth, improve symptoms of HCC patients and extend the life span of advanced HCC patients. One year survival rate of patients with advanced HCC with GJF treatment was improved (GJF group=31\%, control group=4.5\%) [23]. The subcutaneous tumor growth of colon adenocarcinoma could be attenuated by GJF through inhibiting p53 mutations [24]. However, the potential anti-cancer mechanism of GJF is not yet clarified.

ARPP-19 (cAMP-regulated phosphoprotein 19) is a member of the alpha-endosulfine (ENSA) family[25]. It plays a role in tumorigenesis and development through regulating cell proliferation, cell cycle and survival [26-29]. It has been shown that the PP2A is a crucial downstream signaling molecule of ARPP-19 and suppress the growth of tumor[26]. Other research shows that ARPP-19 could regulate the pathogenesis of breast and prostate cancer by vasoactive intestinal peptide (VIP) [27, 28]. Dopamine phosphorylates and induces expression of ARPP-19[27], which plays an indispensable part in the initiation and progression of cancer[29]. Our previous studies demonstrated that elevated ARPP-19 level in HCC tissues and downregulation of ARPP-19 decreased the activation of mitotic substrates, resulting in cell cycle arrest at the G2/M phase in various human HCC cells[30]. These results indicate that ARPP-19 is a potential target of anti-cancer drugs. 
Therefore, in this study, we investigate the effect of GJF on HCC in vivo with HCC xenograft mice model and try to explore its potential mechanism associated with inhibition of tumor proliferation through regulating ARPP-19.

\section{Methods}

\section{Preparation of GJF}

GJF formula is composed of 17 Chinese medicinal herbs: radix pseudostellariae, Panax japonicus C.A.Mey.Var.major(Burkill) C.Y.Wu et K.M.Feng, Atractylodis Macrocephalae, Poria cocos, Salvia miltiorrhiza Bge, Phlegmariurus pulcherrimus, Ma Lan root, ostreagigastnunb, Prunella vulgaris L, Manis pentadactyla, turtle shell, centipede, earthworm, Fructus Akebiae, rhizoma arisaematis, pseudobulbus cremastrae seu pleiones, rhizoma pinelliae, which were mixed with the dosage $15,9,9,12,9,15,15,15$, $15,3,9,9,9,9,6,6$ and $6 \mathrm{~g}$, respectively for the adult daily dose. The dried herbs were purchased from Longhua Hospital, Shanghai University of Traditional Chinese Medicine. The mixed herbs were boiled in $500 \mathrm{~mL}$ of sterile water for $30 \mathrm{~min}$, then the aqueous extracts were filtered and condensed and stored at 4 ${ }^{\circ} \mathrm{C}$ for further animal experiments.

\section{Cell Culture}

HepG2 cells and SMMC 7721 cells were purchased by the Cell Bank of the Chinese Academy of Sciences (Shanghai, China). Cells were cultured in DMEM medium supplemented with $10 \%$ fetal bovine serum (GIBCO, USA), $100 \mathrm{U} / \mathrm{mL}$ penicillin and $100 \mu \mathrm{g} / \mathrm{mL}$ streptomycin (Biowest, Nuaillé, France). The cells were incubated in a humidified atmosphere with $95 \%$ air and $5 \% \mathrm{CO}_{2}$ at $37{ }^{\circ} \mathrm{C}$.

\section{Nude mouse HCC xenograft model and treatment}

Male BALB/c-nude mice (6-week) were purchased from Shanghai Slac Laboratory Animal CO. LTD. The mice were raised in aseptic standard environment with constant temperature $(23 \pm 2 \circ \mathrm{C})$ and controlled light (12 $\mathrm{h}$ light: $12 \mathrm{~h}$ dark). The procedure of the study was approved by Ethics Committee for Research in Animals, Longhua hospital, Shanghai University of Traditional Chinese Medicine. Animal welfare and experimental procedures were administered on the basis of the Guide for the Care and Use of Laboratory Animals.

HepG2 cells were digested and washed with ice-cold PBS. Subsequently, $5 \times 10^{6} \mathrm{HepG} 2$ cells in $200 \mathrm{uL}$ DMEM were injected into the right axillary region of the BALB/c-nude mice. Afterwards, mice were randomly assigned into three groups ( $n=8$ per group). The mice of GJF-L group and GJF-H group received intragastric administration of low or high dose of GJF once a day, respectively (The low dose is calculated according to the standard dose of clinical practice for adult and the equivalent dose of adult and mouse body surface area, and the high dose is twice of the low dose). The control group was 
administrated with normal saline by gavage. The treatments lasted for 4 weeks. The activity of xenograft mice was observed and body weight was measured every four or five days. The length and width of tumors was detected with a vernier caliper every four or five days. The volume of tumor was calculated depending on the following formulas: volume $=a * b^{2} / 2$, " $a$ " is the maximal width and " $b$ " is the maximal orthogonal width[31]. On the 28th day of treatment, all mice were weighed and sacrificed. Then the tumors were excised and weighed. Mice livers and kidneys were removed, then frozen or fixed in $10 \%$ formalin.

For the investigation of survival time, $1 \times 10^{7}$ SMMC 7721 cells were injected into the right axillary region of the BALB/c-nude mice. The tumor-bearing mice were allocated into two groups (Control and GJF-H, $\mathrm{n}=8$ per group) and treated as above description. The death number of mice was recorded in either natural death or euthanasia due to the large tumor (diameter $>15 \mathrm{~mm}$ ), and the survival time was analyzed.

\section{Immunohistochemistry}

For the immunohistochemistry analysis of Ki-67 and ARPP-19, tumor tissues were embedded in paraffin and $4-\mu \mathrm{m}$ thick sections were sliced. Tissue sections were deparaffinized, rehydrated, and subjected to antigen retrieval by immersing sections in citrate buffer $(10 \mathrm{mM}$; $\mathrm{pH} 6.0)$ at $95^{\circ} \mathrm{C}$ for $10 \mathrm{~min}$, then treated with $3 \% \mathrm{H}_{2} \mathrm{O}_{2}$ for 30 min to block endogenous peroxidase activity, and blocked with $2.5 \%$ bovine serum albumin. Subsequently, the sections were incubated with primary antibody against ARPP-19 (Abcam, Cambridge, UK) and antibody against Ki67 (Cell signaling technology, Boston, MA, USA) at $4{ }^{\circ} \mathrm{C}$ overnight. The sections were incubated with the horseradish peroxidase-conjugated secondary antibody (Dako Deutschland $\mathrm{GmbH}$, Hamburg) for $1 \mathrm{~h}$ at room temperature, and brown color was developed with a 3,3'diaminobenzidine chromogenic reagent (DAB) (Dako) and counterstained with hematoxylin (Kehui Biological Technology, Shanghai, China). The stained sections were observed and photographed under a light microscope. The number of Ki67 positive cells per 200× field was counted using ImageJ automated cell counting. The positive expression rates of Ki67 were calculated as follows: positive expression rates of Ki67 (\%) = the number of positive expression cells/the total number of cells $\times 100 \%$.

\section{Histological examination of liver and kidney Tissues}

The liver and kidney sections were stained with H\&E according to the standard methods. In brief, the fresh tissue samples were fixed in $10 \%$ formalin and were embedded in paraffin. The samples were then crosscut into slices of $4 \mu \mathrm{m}$ and were stained with H\&E staining solution (Kehui). Subsequently, the stained sections were observed and photographed under a light microscope (with magnification of 200x).

\section{Western Blot}


The tissues were lysed with RIPA lysis buffer with protease and phosphatase inhibitors for $30 \mathrm{~min}$ on ice. Then, the tissues were centrifuged at $12,000 \mathrm{rpm}$ for $15 \mathrm{~min}$ at $4^{\circ} \mathrm{C}$. The supernatant was collected. Using the BCA method, the protein concentration was detected. Equal amount of protein was resolved by sodium dodecyl sulfate-polyacrylamide gel electrophoresis (SDS-PAGE) and transferred onto the Immobilon-P PVDF membranes (Millipore, Billerica, MA, USA). The membranes were blocked with TBST containing $5 \%$ skimmed milk for $1 \mathrm{~h}$. Then the membranes were incubated with primary antibody against ARPP-19, PCNA (Proteintech, Wuhan, China), Cdc2, phospho-Cdc2, phospho-(Ser) CDKs substrate (Cell Signaling Technology, Boston, MA, USA) or $\beta$-actin (Hua'an biological technology, Hangzhou, China) as internal loading control. After being washed with TBST, the membranes were incubated with secondary antibody (Thermo Scientific, Rockford, IL, USA) for $1 \mathrm{~h}$. Subsequently, the membrane was incubated in a horseradish peroxidase-conjugated goat anti-rabbit or anti-mouse secondary antibody from for $1 \mathrm{~h}$. The signal was visualized by enhanced chemiluminescence HRP substrate (Millipore, Billerica, MA, USA) and acquired by GBOX Chemi XT4 System (Syngene, Cambridge, UK). GeneTools software (Syngene) was used for the quantification.

\section{Statistical Analysis}

In this experiment, the technical statisticians conducted statistical analysis by Statistical Product and Service Solution (SPSS) statistics sofware (IBM, New York, USA). The data were expressed as mean \pm standard deviation (SD). Statistical analyses were carried out using one-way ANOVA, followed by Tukey's post-hoc test to assess the differences between the two groups. The two-tailed Student's t-test was used for the analysis between two groups. $p<0.05$ is regarded statistically significant.

\section{Results}

\section{GJF improved general health state of the HCC tumor- bearing mice and exhibited no toxic effects}

After intragastric administration of GJF for 28 days, compared with model group, favorable effects in the general status, such as more food intake and better mobility were observed in HepG2-xenografted nude mice with GJF treatment. Moreover, as shown in Fig.1, GJF increased the body weight of nude mice in a dose-dependent manner. Furthermore, GJF caused no toxic pathological changes in the tissue structure of liver and kidney in HepG2-xenografted nude mice (Fig.2).

\section{GJF treatment suppressed tumor growth of HCC tumor- bearing nude mice}

We evaluated the anti-cancer effect of GJF on male nude mice with HepG2 xenograft tumor. After 28 days administration of GJF, the volume of tumors was shown to be lowered in GJF formula-treated 
groups when compared with untreated control group. The effect of high dose is more significant $(p<$ 0.01). The tumor weights of mice after treatments with high dose of GJF formula were significantly decreased than those of untreated control mice $(p<0.05)$, and low dose of GJF had the tendency to decrease tumor weight (Fig. 3).

\section{GJF inhibited cell proliferation of HCC tumor}

The Ki67 protein is a cellular marker for cell proliferation and tumor growth. The Ki67 was detected within the cell nucleus by IHC. Compared with the control group, tumor tissue of GJF-H or GJF-L groups showed less Ki67 staining. The positive expression rate of Ki67 is also called Ki67 proliferation index (PI), which is normally incorporated in the evaluating indexes for malignant degree of tumor. Fig.3b showed GJF reduced the Ki67 PI significantly in a dose-dependent manner.

Proliferating cell nuclear antigen (PCNA) is also closely related to DNA synthesis and can be used as an indicator of cell proliferation. The protein level of PCNA was lowered in the tumor treated by GJF obviously (Figure 3c, 3d).

\section{GJF down-regulated ARPP-19 expression of HCC tumor tissues}

The ARPP-19 plays an important role in HCC cell proliferation. Previous research has shown that the major characteristic of ARPP-19 was that ARPP-19 took effect on HCC pathogenesis through decreasing protein levels of phospho-(Ser) CDKs substrates and increasing levels of inactivated cyclin division[30]. To investigate whether GJF could impact the ARPP-19 expression, it was measured by western blot and IHC in the tumor tissues of HepG2-xenografted nude mice. As shown in Figure 4(a) and Figure 4(b), all treatments with GJF inhibited the protein levels of ARPP-19 compared with the control group $(P<0.01)$. Moreover, the effect of GJF depends on the given doses.

\section{GJF inhibited the activation of mitosis-related protein of HCC tumor tissues}

Some studies have shown that a subfamily of cyclin-dependent kinases (Cdks) promote the mammalian cell cycle. Cyclins consists of complicated combinations of Cdks and specific regulatory proteins. Then, Cdk-Cyclin drive the cell forward through the cell cycle [32]. Mitotic substrates measure the cell division. Cdc2 phosphorylate mitotic substrates which regulate the G2/M transition directly. Therefore, we detect the effect of GJF on the level of phosphorylation of Cdc2 and Cdks substrates in the tumor tissues of HepG2-xenografted nude mice by using an antibody recognizing the phospho-serine Cdk consensus motif. As shown in Figure 4(c), all treatments with GJF reduced the protein expression of phosphorylation 
of mitotic substrates $(p<0.05)$. A significantly elevated level of inactivated Cdc2 (phospho-cdc2 (Tyr15)) was also found in GJF groups ( $p<0.05$ ), indicating that GJF could attenuate the activation of Cdc2.

\section{Herbal formula treatment had the tendency of prolonging survival of HCC tumor-bearing mice}

The survival time of mice in the treatment groups were recorded. As shown in Table 1, the mice treated with GJF-H lived for longer period. After tumor cell inoculation, the median survival days of mice in GJF-H group was 41.6 days, which was a bit longer than that in control (37.25 days) group. Although there is no statistical difference, which might be due to less animal number and the limitation of tumor volume leading to short observing period, there is the tendency.

\begin{tabular}{|llllll|}
\hline Group & $\mathbf{N}$ & Mean & Std. Deviation & Minimum & Maximum \\
\hline Control & 8 & 37.25 & 6.155 & 20 & 45 \\
\hline GJF-H & 8 & 41.60 & 5.082 & 31 & 47 \\
\hline
\end{tabular}

Table 1

The survival time of HCC tumor-bearing mice

\section{Discussion}

TCM offers potential for HCC treatment with the fewer adverse effects. In order to guarantee the validity and reliability, evidence-based preclinical and clinical explorations are required. Moreover, elucidation of the underlying pharmaceutical mechanism could benefit the acceptance of TCM application [12-14, 3335].

In the past 50 years, the Department of Oncology, Longhua Hospital, Shanghai University of Traditional Chinese medicine has been devoted to the clinical practice and mechanism exploration of the prevention and treatment of digestive tumors by TCM. According to the TCM pathogenesis theory of liver cancer, "spleen deficiency is the internal basic, qi stagnation, blood stasis, damp heat, toxin and phlegm turbidity are the manifestation characteristics" and clinical experiences, under the leadership of Professor Qiu Jiaxin, the anti-tumor formula "Ganji Fang" has been developed, with the function of "invigorating the spleen and regulating qi, clearing away heat and detoxification, softening and resolving phlegm". Clinical application has shown that GJF can prolong the survival time of patients with advanced liver cancer. Early animal experiments showed that GJF can prevent the occurrence of HCC induced by diethylnitrosamine (DEN)[36]. GJF has cytotoxic effect on HCC cells, and the whole formula is the strongest. Some ingredients, such as atractylodes macrocephala, oyster and rhizoma arisaematis, have anti-mutagenic effect, while atractylodes macrocephala and poria cocos make cells disable to start malignant transformation[23]. In this study, we also find the tumor-inhibiting effect of GJF. The 
subcutaneous HCC tumor volume and weight was obviously down-regulated by GJF treatment dosedependently. Moreover, the xenografted nude mice with GJF treatment had improved food intake, activity and body weight. And the prolonged survival tendency was observed in mice of GJF group. These results indicate the effect of GJF on tumor growth and life quality. In addition, no toxic pathological changes in the tissue of liver and kidney suggested safety of application.

To further explore the anti-tumor mechanism of GJF, Ki67 proliferation index (PI) and PCNA, the proliferation indictor closely related to tumor malignancy were determined. The results showed GJF significantly reduced the $\mathrm{Ki} 67 \mathrm{PI}$ and PCNA level in a dose-dependent manner. The cell cycle is crucial in tumor proliferation and growth. Our previous researches confirmed that ARPP-19 participated in tumor growth of $\mathrm{HCC}[30]$. The molecule plays an important role in the pathogenesis of $\mathrm{HCC}$ by regulating cell cycle[37-40]. Therefore, we measured the expression level of ARPP-19 of the HCC tumor tissues. The IHC and western blot results showed obvious decreased ARPP-19 in GJF group than control dosedependently.

Previous studies have shown the function of ARPP-19 in cell mitosis[38]. Cyclin B-Cdc2 is the universal regulator of mitosis phase of cell cycle. Activated Cyclin B-Cdc2 phosphorylates mitotic CDK substrates to trigger the mitosis. ARPP-19 could be activated by a starter amount of activated Cdc2 through a kinase greatwall $(\mathrm{Gwl})$ or activated directly by Cdc2. In turn, it plays a role in the activation and maintenance of cyclin B-Cdc2 activity[38, 40]. Additionally, it can also inhibit dephosphorylating mitotic substrates during $M$ phase. Therefore, ARPP-19 is involved in the core element of the autoregulatory loop of cyclin BCdc2[39, 41]. In consistence with ARPP-19 expression, we also observed higher level of inactivated Cdc2 (phospho-cdc2) and lower Cdks mitosis substrates in tumor tissues of GJF group than control group. Therefore, the regulation of cell cycle through ARPP-19 is closely associated with the effect of GJF (Figure 7).

\section{Conclusion}

In summary, this study shows the effect of GJF in inhibiting tumor growth of HCC without toxicity, and can improve the quality of life of tumor bearing nude mice. The anti-tumor effect of GJF is related to the inhibition of HCC cell proliferation by arresting cell mitosis through down-regulating ARPP-19. This study provides a scientific basis for the treatment of HCC with TCM. However, the follow-up random clinical control trials are still need to be carried out to confirm the efficacy. In addition, since the multi-target effect of traditional Chinese medicine, the relevant mechanism research also needs to be further explored in order to promote its application in HCC treatment.

\section{Abbreviations}

HCC: Hepatocellular carcinoma; GJF: Ganji Fang; TCM: Traditional Chinese Medicine; GJF-H group: high dose of GJF; GJF-L group: low dose of GJF; ARPP-19: cAMP-regulated phosphoprotein 19; Cdc2: cyclin division cycle 2; DCR: disease control rate; SYY: Songyou Yin; EMT: epithelial-mesenchymal transition; 
HQT: Huang-qin-tang; LV: leucovorin; ENSA: alpha-endosulfine; VIP: vasoactive intestinal peptide; SDSPAGE: sodium dodecyl sulfate-polyacrylamide gel electrophoresis; SPSS: Statistical Product and Service Solution; SD: standard deviation; PI: proliferation index; PCNA: Proliferating cell nuclear antigen; Cdks: cyclin-dependent kinases; DEN: diethylnitrosamine; Gwl: greatwall

\section{Declaration}

\section{Authors' contributions}

SH and ZP designed the study. ZC, TK performed the major studies. ZY contributed to part of the animal experiments. $\mathrm{YL}$ and $\mathrm{LY}$ analyzed and interpreted the data. $\mathrm{YJ}$ provided advices on study design and the formula application. ZC has drafted the work and SH substantively revised it. All authors read and approved the final manuscript.

\section{Acknowledgements}

Not applicable.

\section{Competing interests}

The authors declare that they have no competing interests.

\section{Availability of data and materials}

All data generated or analysed during this study are included in this published article and. are available from the corresponding author on reasonable request.

\section{Consent for publication}

Not applicable.

\section{Ethics approval and consent to participate}

All experimental procedures in mice were approved by the Experimental Animal Care and Use Committee of Longhua Hospital, Shanghai University of Traditional Chinese Medicine.

\section{Funding}


This study was partially supported by the National Natural Science Foundation of China (81202667, 81703867).

\section{References}

1. Rebecca L, Siegel MPH, Kimberly D, Miller MPH, Ahmedin, J. Cancer statistics, 2016. Ca A Cancer Journal for Clinicians. 2016; 66: 7-30.

2. Chen W, Zheng R, Baade PD, Zhang S, Zeng H, Bray F, Jemal A, Yu XQ, He J. Cancer statistics in China, 2015. Ca Cancer J Clin. 2016;66(2):115-32.

3. Ogawa K. Molecular pathology of early stage chemically induced hepatocarcinogenesis. Pathology International. 2009;59(9):605-22.

4. Thorgeirsson S, Grisham J. Molecular pathogenesis of human hepatocellular carcinoma. Toxicology. 2002;181(4):43-7.

5. Zeng H, Zhen R, Guo Y, Zhang S, Zou X, Wang N, Zhang L, Tang J, et al.Cancer survival in China, 2003-2005: A population-based study. International Journal of Cancer. 2015;136(8):1921-30.

6. Schwartz M, Roayaie S, Konstadoulakis M. Strategies for the management of hepatocellular carcinoma. Nature Clinical Practice Oncology. 2007;4(7):424-32.

7. Andreou A, Vauthey J, Cherqui D, Zimmit G, Ribero D, Truty MJ, et al. Improved Long-Term Survival after Major Resection for Hepatocellular Carcinoma: A Multicenter Analysis Based on a New Definition of Major Hepatectomy. Journal of Gastrointestinal Surgery. 2013;17(1):66-77.

8. Xie B, Wang DH, Spechler SJ. Sorafenib for Treatment of Hepatocellular Carcinoma: A Systematic Review. Digestive Diseases \& Sciences. 2012;57(5):1122-9.

9. Carr BI. Hepatocellular carcinoma: Current management and future trends. Gastroenterology. 2004;127(S1): 218-24.

10. Ponziani FR, Bhoori S, Germini A, Bongini M, Flores M, Sposito C, et al. Inducing tolerability of adverse events increases sorafenib exposure and optimizes patient $\backslash$ "s outcome in advanced hepatocellular carcinoma. Liver International Official Journal of the International Association for the Study of the Liver. 2016;36(7):1033-42.

11. Roberto I, Maurizio P, Emanuele R, Eleonora A, Matteo G, Lucia C, et al. TACE with degradable starch microspheres (DSM-TACE) as second-line treatment in HCC patients dismissing or ineligible for sorafenib. European Radiology. 2018; 29(5): 1-8.

12. Hu B. Traditional Chinese medicine for prevention and treatment of hepatocarcinoma: From bench to bedside. World Journal of Hepatology. 2015;7(9).

13. Zheng T, Que Z, Jiao L, Kang Y, Xu L. Herbal formula YYJD inhibits tumor growth by inducing cell cycle arrest and senescence in lung cancer. Sci Rep. 2017;7(1):4984.

14. Lina, Pang, Shuyan, Han, Yanna, Jiao, et al. Bu Fei Decoction attenuates the tumor associated macrophage stimulated proliferation, migration, invasion and immunosuppression of non-small cell 
lung cancer, partially via IL-10 and PD-L1 regulation. International Journal of Oncology. 2017; 51(1): 25-38.

15. Qi F, Li A, Inagaki Y, Gao J, Tang W. Chinese herbal medicines as adjuvant treatment during chemoor radio-therapy for cancer. Bioscience Trends. 2010;4(6):297-307.

16. Ning $Y$, Ying $X$, Wang C. Bu-Zhong-Yi-Qi Decoction, the Water Extract of Chinese Traditional Herbal Medicine, Enhances Cisplatin Cytotoxicity in A549/DDP Cells through Induction of Apoptosis and Autophagy. Biomed Research International. 2017;2017:1-9.

17. Zhao J, Liu L, Zhang Y, Wan Y, Hong Z . The herbal mixture Xiao-chai-hu Tang (xcht) induces apoptosis of human hepatocellular carcinoma Huh7 cells in vitro and in vivo. African Journal of Traditional, Complementary and Alternative medicines. 2017; 14:231- 241.

18. Xiong W, Ren Z, Qiu S, Sun H, Wang L. Residual hepatocellular carcinoma after oxaliplatin treatment has increased metastatic potential in a nude mouse model and is attenuated by Songyou Yin. Bmc Cancer. 2010; 10: 219.

19. Zheng S, Jia Q, Shen H, Xu X, Ling J, Jing C, et al. Treatment with the herbal formula Songyou Yin inhibits epithelial-mesenchymal transition in hepatocellular carcinoma through downregulation of TGF- $\beta 1$ expression and inhibition of the SMAD2/3 signaling pathway. Oncology Letters. 2017;13: 2309-2315.

20. Chu E. Wedding Rigorous Scientific Methodology and Ancient Herbal Wisdom to Benefit Cancer Patients: The Development of PHY906. Oncology. 2018;32(2):e20-e7.

21. Lam W, Jiang Z, Guan F, Huang X, Hu R, Wang J, et al. PHY906(KD018), an adjuvant based on a 1800-year-old Chinese medicine, enhanced the anti-tumor activity of Sorafenib by changing the tumor microenvironment. Scientific Reports. 2015;5:9384.

22. Kummar S, Copur MS, Rose M, Wadler S, Stephenson J, O'Rourke M, Brenckman W, Tilton R, Liu SH, Jiang Z, Su T, Cheng YC, Chu E. A Phase I Study of the Chinese Herbal Medicine PHY906 as a Modulator of Irinotecan-based Chemotherapy in Patients with Advanced Colorectal Cancer. Clin Colorectal Cancer. 2011;10(2):85-96.

23. Qiu J, Yang J. Clinical observation and experimental study of Jianpi Liqi, Qingre Jiedu and Rujian Huatan prescription in treating advanced liver cancer . Chin J Integrated Tradit Chin West Med. 1987; (260):275-7 (in Chinese).

24. Zhao A, Yang J, Xiao Z. The Effect of SRRS Prenventing P 53 Gene from Mutation. Cancer Research on Prevention \& Treatment. 1999.

25. Horiuchi A, Williams KR, Kurihara T, Nairn AC, Greengard P. Purification and CDNA cloning of ARPP16, a cAMP-regulated phosphoprotein enriched in basal ganglia, and of a related phosphoprotein, ARPP-19. Journal of Biological Chemistry. 1990;265: 9476-9484.

26. Eichhorn PJ, Creyghton MP, Bernards R. Protein phosphatase 2A regulatory subunits and cancer. Biochim Biophys Acta. 2009;1795(1):1-15

27. Girault JA, Shalaby IA, Rosen NL, Greengard P. Regulation by cAMP and vasoactive intestinal peptide of phosphorylation of specific proteins in striatal cells in culture. Proceedings of the National 
Academy of Sciences of the United States of America. 1988;85(20):7790-4.

28. Milena V, Violeta D, Milivoj D, Branch DR, Nevena V, Sakarellos-Daitsiotis MM, et al. Physical Activity and Natural Anti-VIP Antibodies: Potential Role in Breast and Prostate Cancer Therapy. Plos One. 2011;6(11):e28304-.

29. Sachlos E, Risueno RM, Laronde S, Shapovalova Z, Lee JH, Russell J, et al. Identification of Drugs Including?a Dopamine?Receptor Antagonist that Selectively Target Cancer Stem Cells. Cell. 2012;149(6): 1284-1297.

30. Song S, Pan J, Liu Y, Wen H, Wang L, Cui J, et al. Increased ARPP-19 expression is associated with hepatocellular carcinoma. International Journal of Molecular Sciences. 2015.

31. Wang L, Tang ZY, Qin LX, Wu XF, Sun HC, Xue Q, Ye SL. High-Dose and Long-Term Therapy With Interferon-Alfa Inhibits Tumor Growth and Recurrence in Nude Mice Bearing Human Hepatocellular Carcinoma Xenografts With High Metastatic Potential. Hepatology. 2000;32(1):43-8.

32. Schwartz GK, ; Shah MA. Targeting the Cell Cycle: A New Approach to Cancer Therapy. Journal of Clinical Oncology. 2005;23(36):9408-21.

33. Penglong, Wang, Gaimei, She, Yanan, Yang, et al. Synthesis and Biological Evaluation of New Ligustrazine Derivatives as Anti-Tumor Agents. Molecules. 2012.

34. Yang X-B, Wu W-y, Long S-q, Deng H, Pan Z-Q, He W-F, et al. Fuzheng Kang'ai decoction combined with gefitinib in advanced non-small cell lung cancer patients with epidermal growth factor receptor mutations: study protocol for a randomized controlled trial. Trials. 2015;16(1):146.

35. Li W, Chen C, Saud SM, Geng L, Zhang G, Liu R, et al. Fei-Liu-Ping ointment inhibits lung cancer growth and invasion by suppressing tumor inflammatory microenvironment. Bmc Complementary \& Alternative Medicine. 2014;14(1):153.

36. Qiu J, Yang J, Tang L, Jia Y, Cao L, Wang N, Zuo J, Chen F, Gao W, Zhang W, Li H, Shen k. The effect of traditional Chinese medicine Jianpi Liqi, Qingre Jiedu and Rujian Huatan prescription on liver cancer induced by diethylnitrosamine in rats. Chin $\mathrm{J}$ Integrated Tradit Chin West Med. 1988(710):734-5 (in Chinese).

37. Girault J, Horiuchi A, Gustafson E, Rosen N, Greengard P. Differential expression of ARPP-16 and ARPP-19, two highly related cAMP- regulated phosphoproteins, one of which is specifically associated with dopamine-innervated brain regions. Journal of Neuroscience the Official Journal of the Society for Neuroscience. 1990;10(4):1124-33.

38. Gharbi-Ayachi A, Labbe J-C, Burgess A, Vigneron S, Strub JM, Brioudes E, et al. The Substrate of Greatwall Kinase,Arpp19, Controls Mitosis by Inhibiting Protein Phosphatase 2A. Science. 2010;330(6011):p.1673-7.

39. Okumura E, Morita A, Wakai M, Mochida S, Hara M, Kishimoto T. Cyclin B-Cdk1 inhibits protein phosphatase PP2A-B55 via a Greatwall kinase-independent mechanism. Journal of Cell Biology. 2014;204(6):881-9.

40. Lorca T, Castro A. The Greatwall kinase: a new pathway in the control of the cell cycle. Oncogene. 2013;32(5):537-43. 
41. Dupre A, Buffin E, Roustan C, Nairn AC, Jessus C, Haccard O. The phosphorylation of ARPP19 by Greatwall renders the auto-amplification of MPF independently of PKA in Xenopus oocytes. Journal of Cell Science. 2013;126(17):3916-26.

\section{Figures}

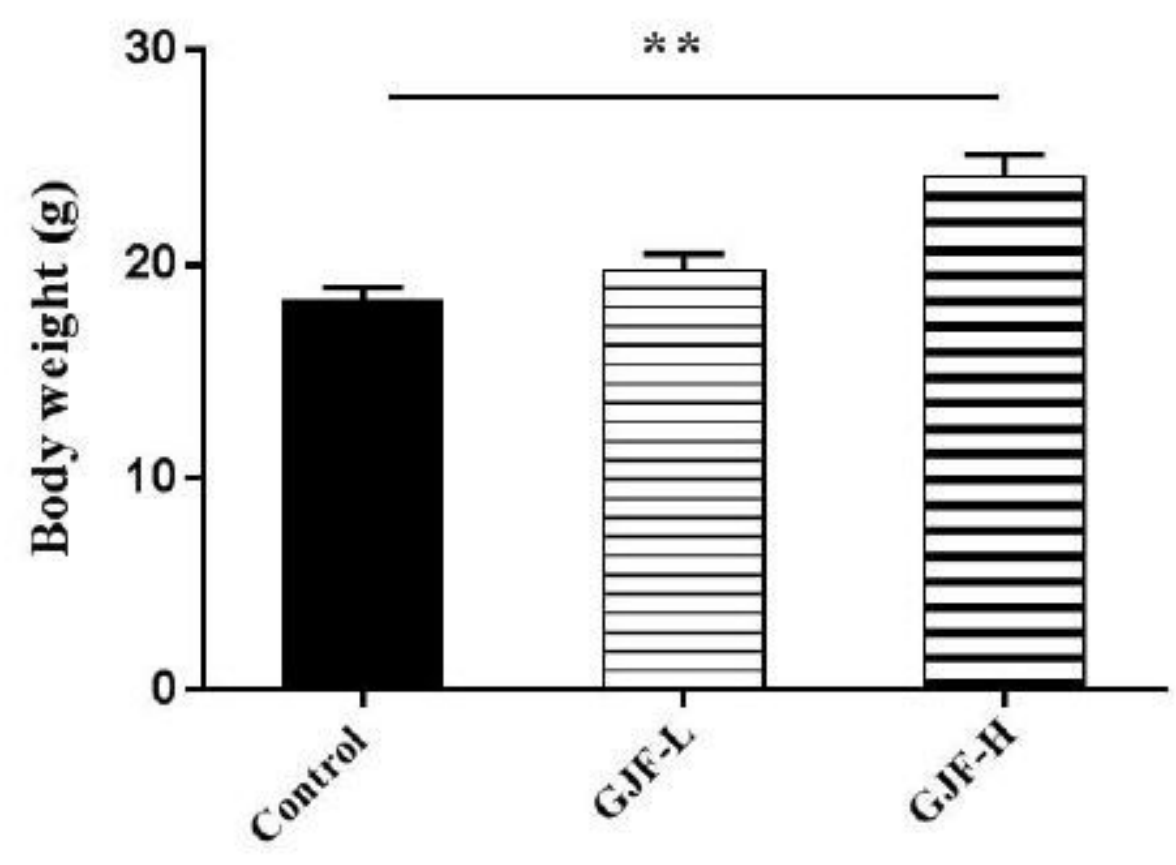

Figure 1

GJF increased the body weight of HCC tumor-bearing nude mice. Data represented mean $\pm S D, n=8$, ** $p$ $<0.01$ vs. control. 
a

\section{Control}

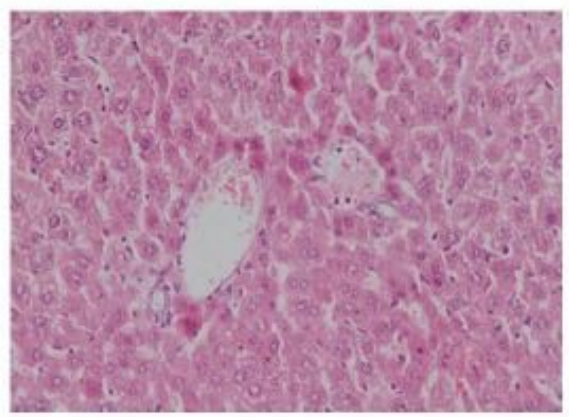

b

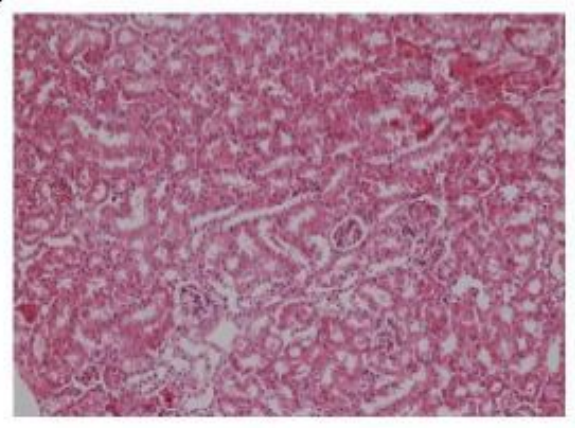

GJF-L
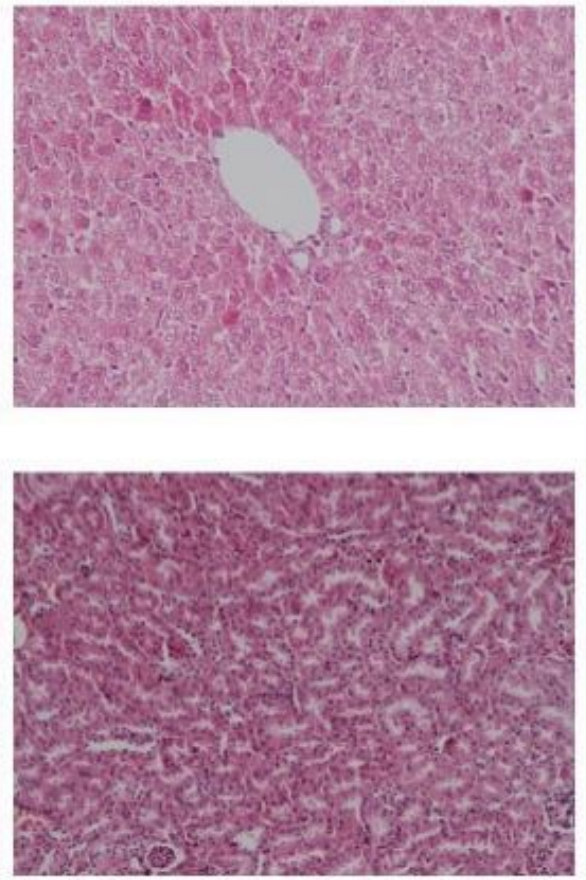

GJF-H
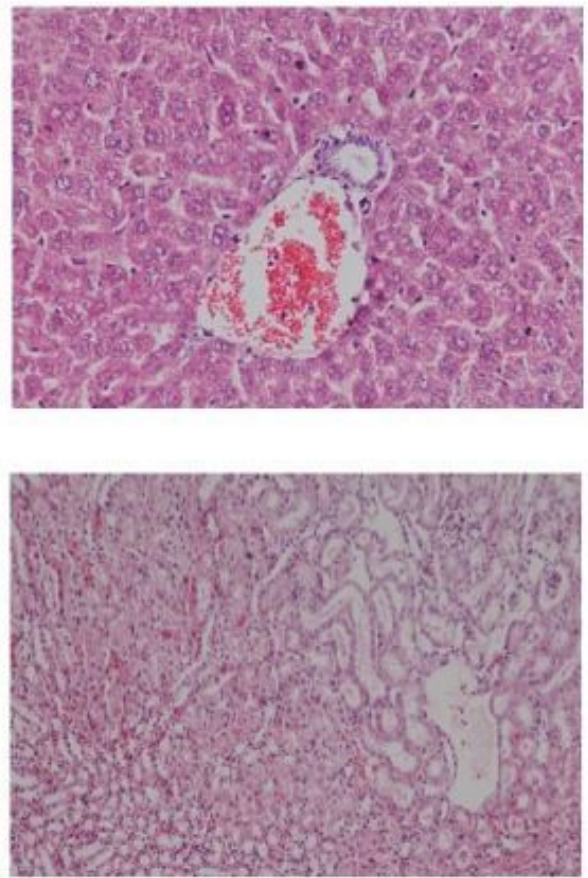

Figure 2

GJF caused no toxic effects for liver and kidney of HCC tumor-bearing nude mice. a Representative HE staining of liver tissue (HE, 200x). b Representative HE staining of kidney tissue (HE, 200x). 
a

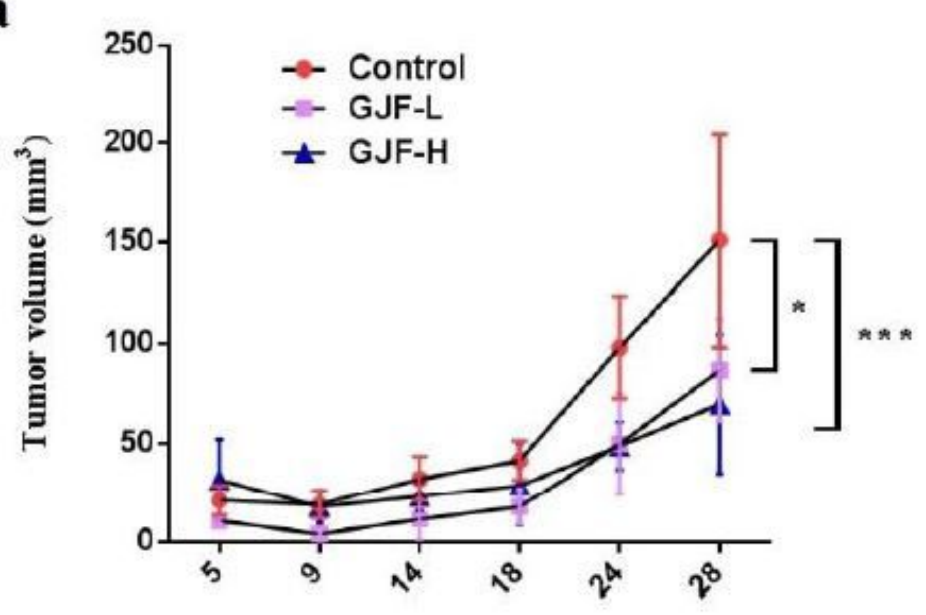

c

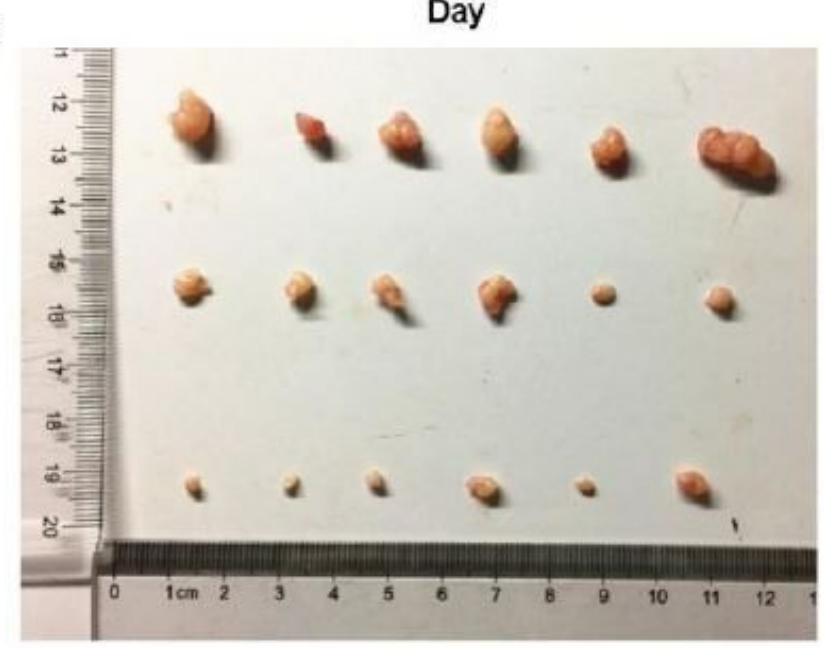

b

\section{Control G.JF-L G.JF-H}

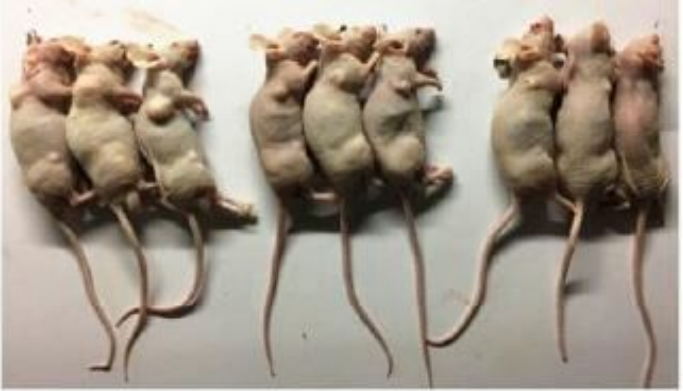

d

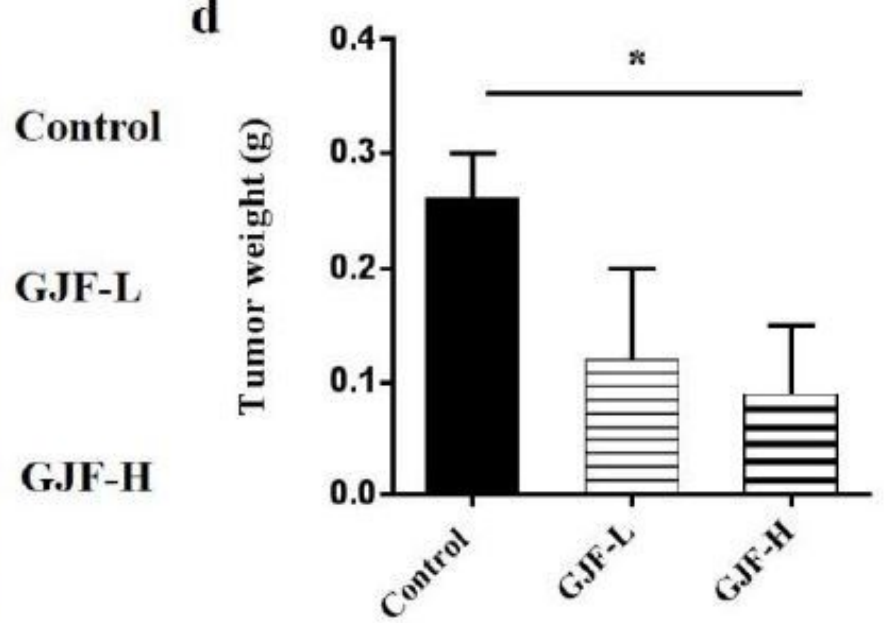

Figure 3

GJF inhibited tumor growth in HCC tumor-bearing nude mice. a The tumor volumes measured during treatment. b The observation of the subcutaneous tumor. c Representative excised tumors from HepG2xenografted nude mice. $d$ The comparison of tumor weight of 3 groups. Data represented mean \pm SD,

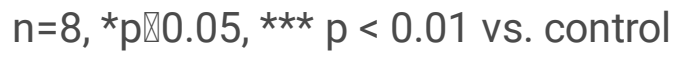


a

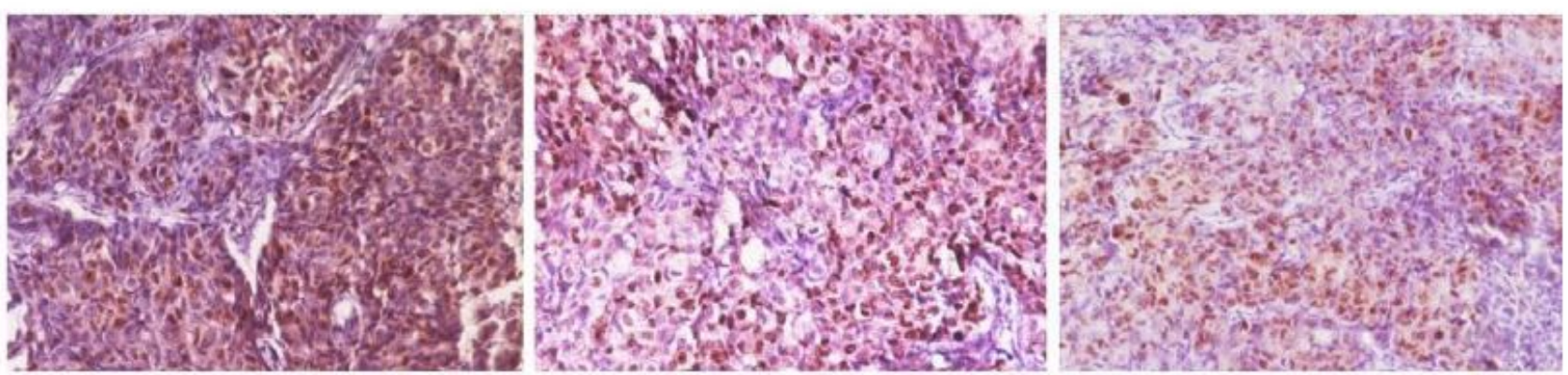

b

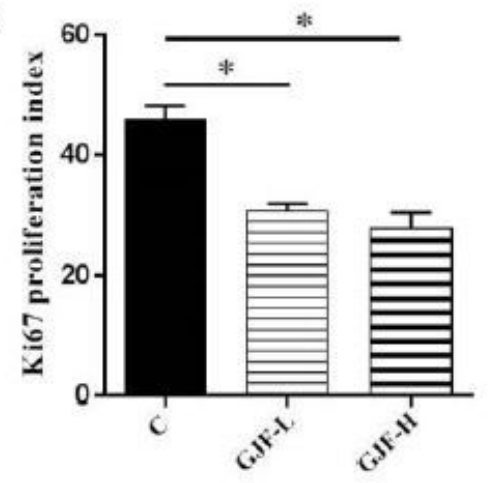

c

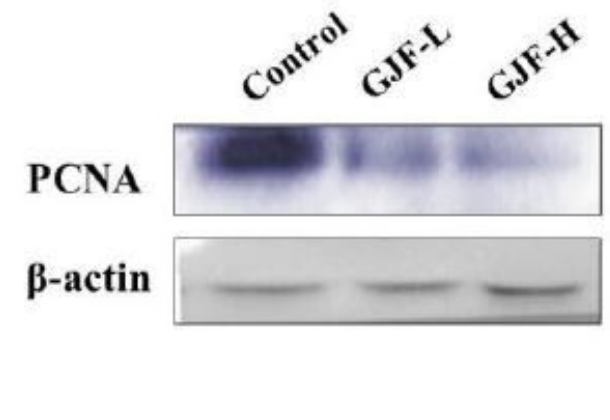

d

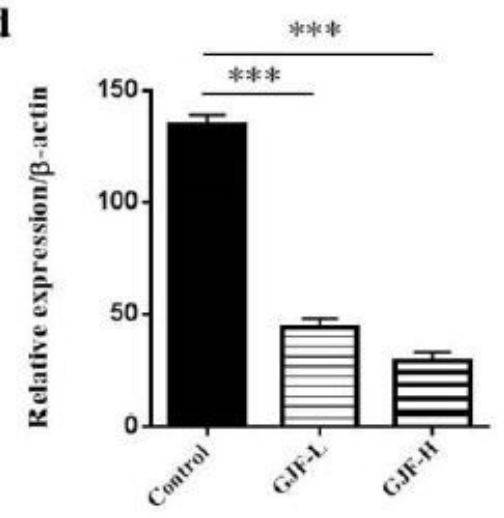

Figure 4

GJF reduced tumor cell proliferation in vivo. a Representative IHC staining of Ki67 (magnification 200x). b Comparison of the Ki67 proliferation index (PI) of three groups, $n=8$. c The PCNA protein levels of tumor tissues analyzed by western blot, with $\beta$-actin serving as an internal reference. $d$ Quantification of data in $c, n=4$. All data were shown as mean $\pm S D, * p<0.05$, ${ }^{* \star} p<0.001$ vs. control. 
a

Control

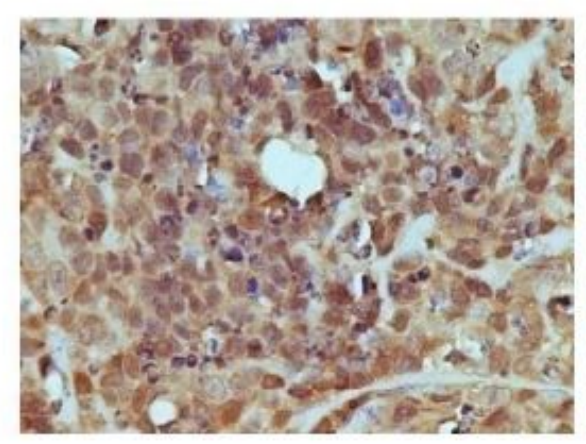

b

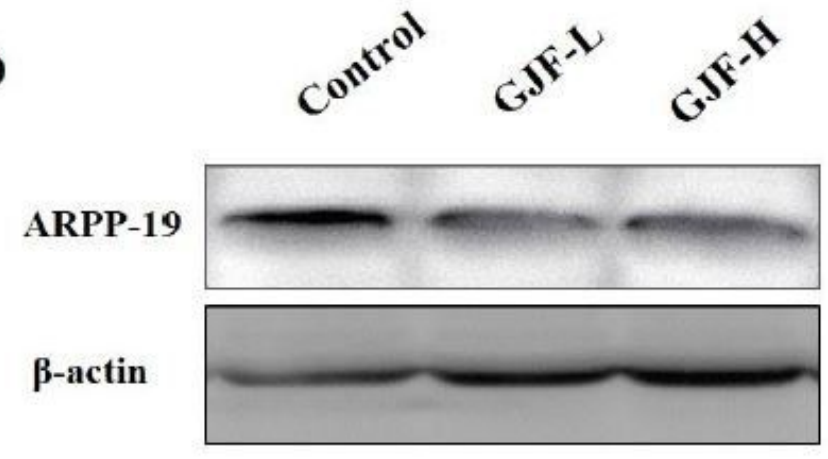

GJF-L

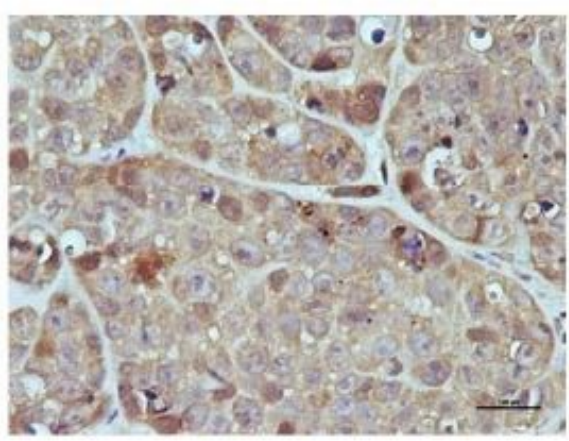

GJF-H
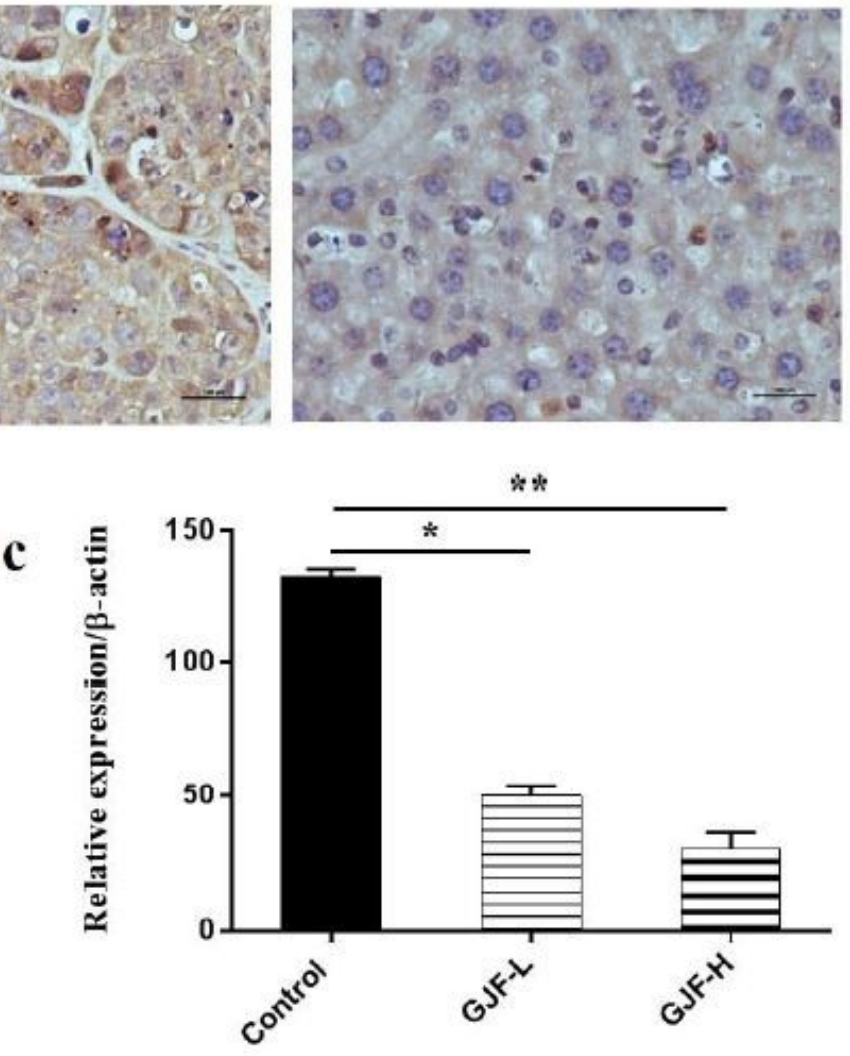

\section{Figure 5}

GJF reduced ARPP-19 protein expression in tumor tissues. a IHC stain showed the ARPP-19 expression (magnification 400x). b Effect of GJF on relative expression of ARPP-19 protein by Western blot, with $\beta$ actin serving as an internal reference. c Quantification of data in b. Data represented mean $\pm S D, n=4$, $* p<0.05$ and ${ }^{*} \mathrm{p}<0.01$ vs. control. 
a

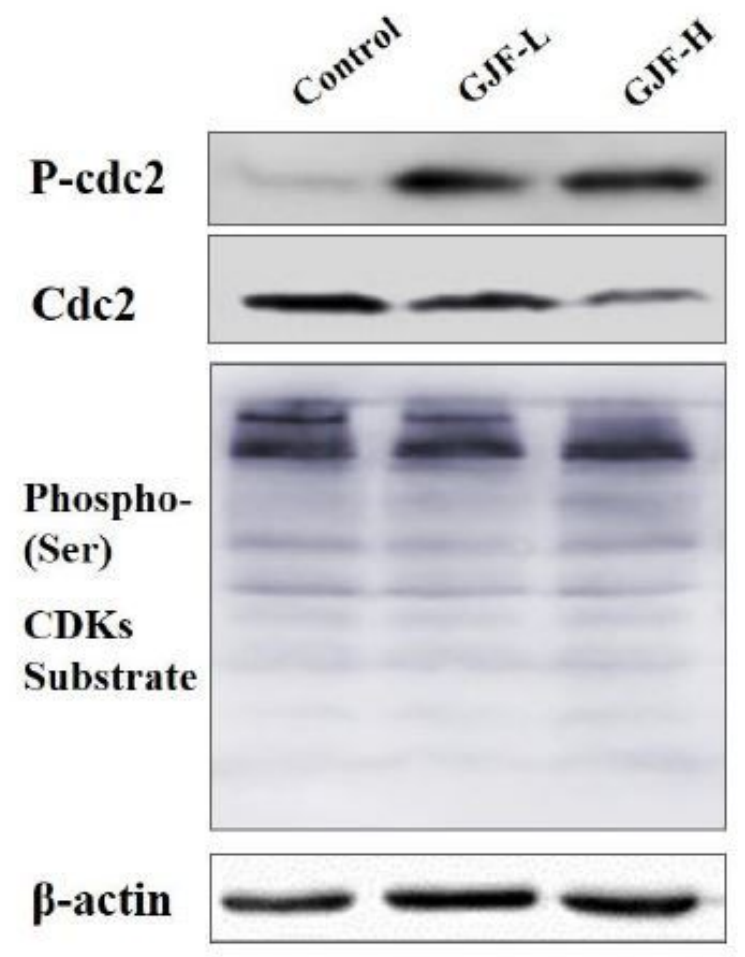

b
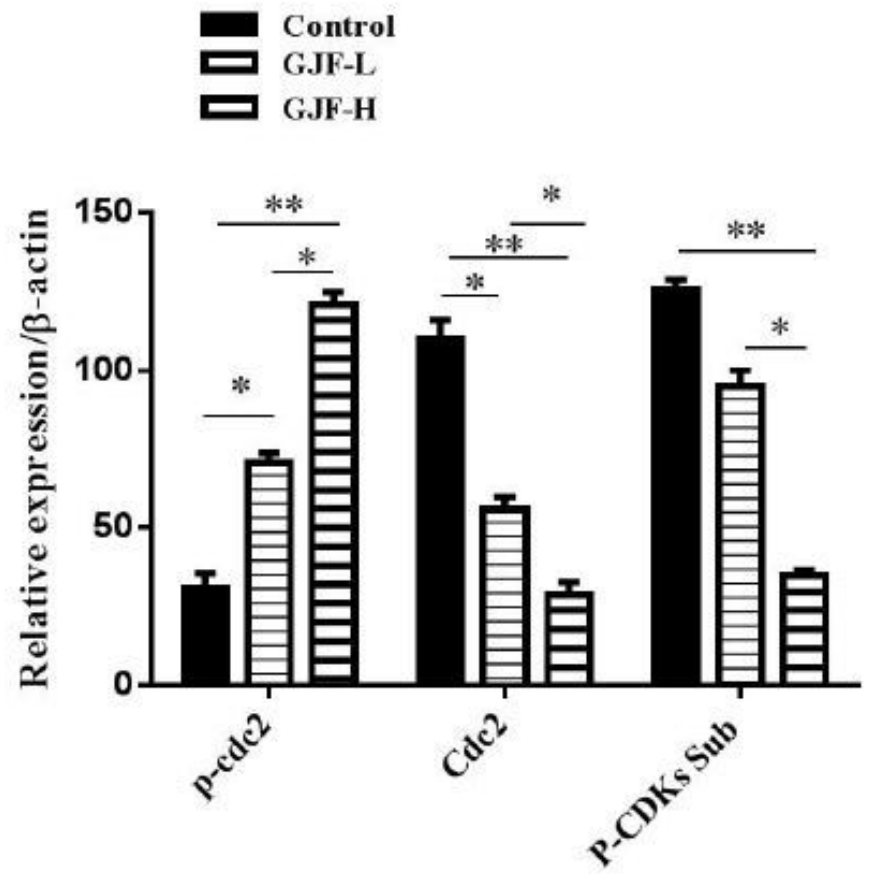

Figure 6

GJF regulated activation cyclin-dependent kinases and phosphorylated mitotic substrates. a Representative blot image of Cdks substrates and Cdc2 protein expression and activation of tumor tissues analyzed by Western blot, with $\beta$-actin serving as an internal reference. b Quantification of data in $b$ analyzed by using densitometry. Data represented mean $\pm S D, n=4, * p<0.05$ and $* * p<0.01$ vs. control. 


\section{Ganji Fang}

Activation

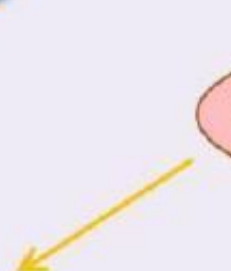

Cyclin B/Cdc2

ARPP-19

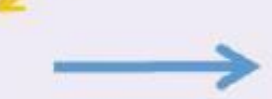

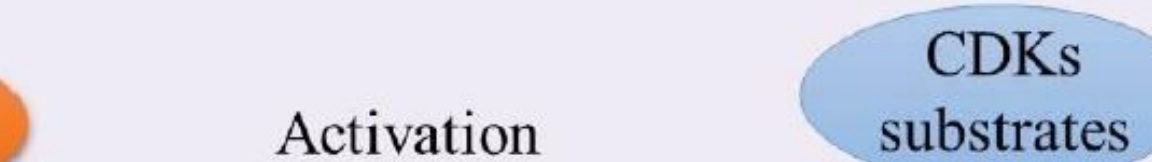

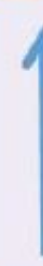

Cyclin B/P-Cdc2 substrates

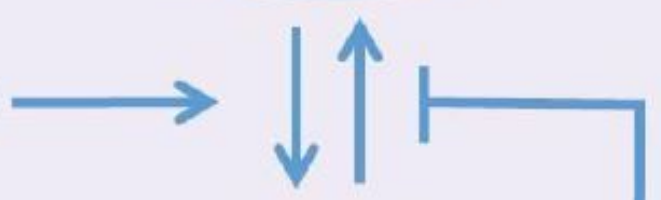

Activation

P-CDKs

substrates

$\downarrow$

Mitosis

\section{Figure 7}

Sketch of the effect of GJF on tumor cell proliferation through regulating ARPP-19 promoted cell mitosis. Activation of cyclin B-Cdc2 is important to trigger cell mitosis. The small starter amount of active cyclin B-Cdc2 could activate ARPP-19, which in turn helps maintain activation of cyclin B-Cdc2. In addition, ARPP-19 plays a role in preventing inactivation of the substrates of Cdc2. Gangji Fang could downregulate ARPP-19 to arrest cell mitosis, thereby inhibit cell proliferation. 